\title{
Transparent conducting oxides for active hybrid metamaterial devices
}

\author{
Martina Abb \\ SEPnet and the Department of Physics and Astronomy, University of Southampton, \\ Highfield, Southampton SO17, 1BJ, United Kingdom
}

\section{Borja Sepúlveda}

Nanobiosensors and Bioanalytical Applications Group, Research Center on Nanoscience and Nanotechnology (CSIC) \& CIBER-BBN, 08193 Bellaterra, Barcelona, Spain

\section{Harold M. H. Chong}

Department of Electronic and Computer Science, University of Southampton, Highfield, Southampton SO17, 1BJ, United Kingdom

\section{Otto L. Muskens}

SEPnet and the Department of Physics and Astronomy, University of Southampton, Highfield, Southampton SO17, 1BJ, United Kingdom

E-mail: o.muskens@soton.ac.uk

\begin{abstract}
We present here a study of the combined nonlinear response of plasmonic antenna - transparent conducting oxide hybrids for activation of metamaterial devices. Nanoantenna layers consisting of randomly positioned gold nanodisk dimers are fabricated using hole-mask lithography. The nanoantenna layers are covered with a 20$\mathrm{nm}$ thin layer of transparent conducting oxide (TCO). Next to indium-tin oxide (ITO) produced using sputter coating, we investigate the response of atomic-layer deposited aluminium-doped zinc oxide (AZO). We show that our results are in agreement with the hypothesis of fast electron-mediated cooling, facilitated by the ohmic interface between the gold nanodisks and the TCO substrate, which appears a universal mechanism for providing a new hybrid functionality to active metamaterial devices.
\end{abstract}

PACS numbers: 78.67.bf, 42.65.Pc, 42.25.Fx, 78.47.J-

Keywords: Plasmonic, Nanoantenna, Metamaterial, Nonlinear, Ultrafast 


\section{Introduction}

Recently there has been increasing interest in alternative materials for application in plasmonics, metamaterials, and transformation optics $[1,2]$. This interest has been fuelled by the desire to achieve more flexibility in design and active control of these phenomena [3]. Apart from optimization of the optical response, combining plasmonic materials with electric, magnetic, or other functionalities is of considerable interest for interfacing of different types of information carriers. The enormous potential of plasmonics and metamaterials for devices is a strong motivation to explore new routes toward active control and switching devices.

Plasmon-induced carrier injection effects have been receiving interest recently for their potential in photodetectors and energy harvesting $[4,5,6]$. Recently, we have shown that picosecond hot-carrier injection can be used to sensitize the optical nonlinearity of a dielectric substrate consisting of the transparent conductor ITO [7]. Transparent conducting oxides (TCOs) are a class of metal oxides which combine a good electronic dc-conductivity with a high optical transparency in the visible and nearinfrared spectral window. Metal oxides are a particularly interesting materials system for integration with plasmonics because of the recent interest in oxide electronic devices $[8,9]$. The optical response of TCOs is governed by free electrons with a density that can be controlled through the addition of n-type dopants. The optical response follows the Drude model, resulting in the dielectric function given by

$$
\tilde{\epsilon}_{\mathrm{ITO}}(\omega)=\tilde{\epsilon}_{\mathrm{exp}}(\omega)-\left(\frac{\omega_{\mathrm{pl}}}{\omega}\right)^{2} \frac{1}{1+i\left(\omega \tau_{D}\right)^{-1}},
$$

where $\omega_{\mathrm{pl}}=\sqrt{N e^{2} / \epsilon_{0} m^{*}}$ denotes the plasma frequency, with $N$ the free electron density, $m^{*}$ the effective electron mass in the band, and $\tau_{D}$ the plasmon relaxation time. For noble metals, $N \simeq 10^{22} \mathrm{~cm}^{-3}$ and their bulk plasmon frequency is located in the uv-range. However, transparent conductors have free-carrier densities in the range $10^{20}-10^{21} \mathrm{~cm}^{-3}$, resulting in a bulk plasma frequency $\omega_{\mathrm{pl}}$ of TCOs in the near-infrared part of the spectrum. For frequencies below $\omega_{\mathrm{pl}}$ the dielectric function is negative and the free-electron gas has a metallic response, i.e. high reflectivity and absorption. For frequencies higher than $\omega_{\mathrm{pl}}$, the free electrons cannot follow the oscillating electric field and therefore the TCO is transparent and has a positive dielectric constant.

In this work, we explore the response of active metamaterial layers consisting of plasmonic antennas covered with a thin layer of TCO. We exploit the high carrier density of the TCO, with correspondingly high dc-conductivity, to enable a fast, picosecond injection scheme as shown schematically in Fig. 1. This scheme was put forward in Ref. [7] to explain our results on single antenna-ITO hybrids and is based on pulsed excitation in the visible using a picosecond laser. Because of the high transparency of the TCO, light-matter interaction takes place primarily in the gold nanoantenna through generation of hot carriers via plasmonic excitation. We hypothesize that the good ohmic contact between gold and TCO subsequently allows fast spill-out of these hot electrons on a subpicosecond time scale, i.e. faster than the electron-phonon relaxation 
time. As the Coulomb screening length in metals and transparent conductors is less than $1 \mathrm{~nm}$, charge imbalance by hot-electron spill-out will be rapidly neutralized by carriers flowing back into the antenna, therefore it is unlikely that significant changes in carrier density occur as a result of this fast electronic process. The main consequence of the fast electronic heat transport is that energy is deposited primarily in the substrate lattice instead of in the gold nanoantenna, as would be the case for antennas on an insulating substrate. In Ref. [7] this was evidenced by an absence of vibrational modes in the nanoantenna-ITO hybrid. The rapid energy deposition in the substrate produces a localized temperature rise, increased carrier mobility and thus a concomitant net diffusion of hot carriers away from the nanoantenna. The resulting space charge is equivalent to the photo-dember effect in semiconductors [10]. The dielectric response of the TCO follows changes in the local carrier density according to Eq. (1), which acts back on the antenna to produce a shift of the plasmonic modes.

Here, we investigate the antenna-TCO hybrid response using random antenna arrays produced by hole-mask lithography over a large surface area. Hole-mask lithography is a versatile fabrication method which can be used to produce uniform plasmonic and metamaterial layers over a large surface area $[11,12,13,14]$. The resulting layers allow us to produce hybrid antenna-TCO devices in a different plasmonic system, showing that this is a universal approach for producing active metamaterials. We extend our results on sputtered ITO by studying the effect using a thin layer of AZO obtained by Atomic Layer Deposition. ZnO-based materials are of enormous interest for their favorable electronic, piezoelectric, and thermal properties for applications in novel electronic devices [15]. Atomic Layer Deposition results in smooth layers of much higher quality than conventional sputtering methods, which is favorable for obtaining reproducible devices.

\section{Method}

Planar plasmonic nanoantennas were fabricated using hole-mask colloidal lithography following the method described in Ref. [11]. In short, colloidal polystyrene spheres were deposited in a monolayer and used as a template for developing a random array of holes in a PMMA resist. Subsequently, gold was evaporated sequentially at very small angles to carefully control both particle form and separation between nanodisks.

For activation of the nanodisk dimers, we investigate transparent conducting oxide layers deposited by different techniques. Indium Tin Oxide (ITO) was deposited using r.f. sputtering of an ITO target (Indium 90\% Tin 10\% from Kurt J. Lesker Company) in an oxygen/argon plasma of mixing ratio 1:136 at a temperature of $490^{\circ} \mathrm{C}$. The deposition was optimized to achieve sheet resistances of $40-50 \Omega / \mathrm{sq}$ for a $200 \mathrm{~nm}$ film of good transparency.

Al-doped $\mathrm{ZnO}$ (AZO) films were produced using Atomic Layer Deposition (ALD) at a temperature of $150^{\circ}$. The $\mathrm{AZO}$ is formed through a layer-by-layer structure consisting of a $\mathrm{ZnO}$ matrix and $\mathrm{Al}_{2} \mathrm{O}_{3}$ dopant layers, with a mixing ratio of 20 cycles of $\mathrm{ZnO}$ 


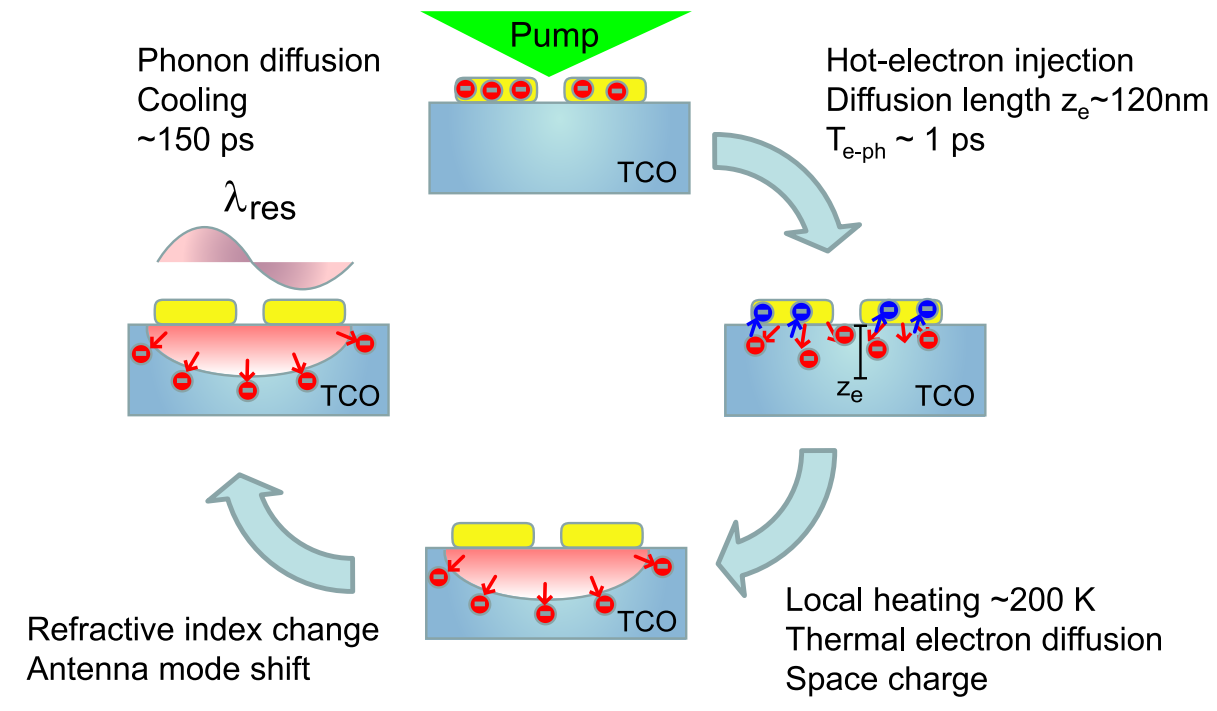

Figure 1. Principle of plasmon-sensitized nonlinear response of the antenna-TCO hybrid produced by plasmonic-induced hot electron injection from the antenna into the TCO substrate, as hypothesized from results of Ref. [7].

and 1 cycle of $\mathrm{Al}_{2} \mathrm{O}_{3}$ for each supercycle, as proposed for optimal resistivity in [16]. ALD is by far the best controlled of the two processes with its controlled growth of single atomic layers, guaranteeing large-area film deposition conformality due to the self-limiting and surface-saturated reaction [17]. Additional advantages include the low deposition temperature and minute control of doping concentration by adjusting the cycle ratio.

Linear and time-resolved nonlinear spectra were collected using a combined transmission and reflection microscope setup based on a broadband supercontinuum light source with two synchronized outputs (Fianium SC450-Dual). The supercontinuum spectrum, covering the visible and infrared spectral range from $0.5-2.0 \mu \mathrm{m}$, was frequency filtered using a double-prism subtractive mode monochromator, where a translating slit was used to select a band of 10-20 nm spectral width. The beam was focused onto the layer through the glass substrate using a long-working distance infrared microscope objective (Mitutoyu NIR-Plan APO, 100×, N.A. 0.5). Reflected light was collected through the same microscope objective and reflected to a Si-photodiode. Transmitted light was collected using an aspheric lens (Edmund Optics, N.A. 0.68) and focused onto a second Si photodiode. The beam was modulated at $0.8 \mathrm{kHz}$ using an optical chopper, lock-in amplification was used for simultaneous acquisition of both reflected and transmitted intensity.

For pump-probe spectroscopy, we used the synchronized output of the ytterbium fibre amplifier, producing pulses of 4 ps duration at a repetition rate of $40 \mathrm{MHz}$. The $1060 \mathrm{~nm}$ output of the laser was frequency doubled using a $5 \mathrm{~mm} \mathrm{KTP}$ crystal to a wavelength of $530 \mathrm{~nm}$. The beam was focused onto the front side of the sample through the aspheric lens in order to maximize the delivered power and to achieve independent control of the focal distance of the pump and probe beams. A translation 


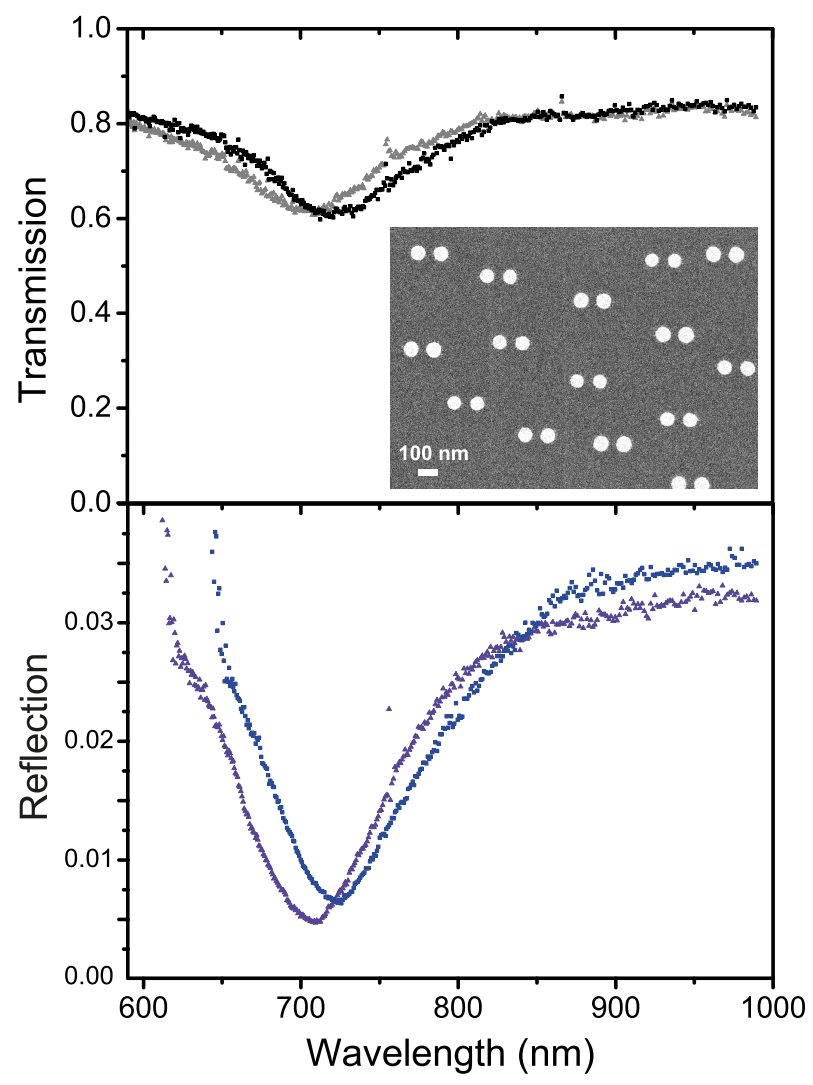

Figure 2. Transmission (top) and reflection (bottom) spectra of gold dimer layer in absence of TCO. We observe a dip in the spectra for both reflection and transmission, which is redshifted for the longitudinal polarization (black in transmission and blue in reflection) with regard to the transverse polarization (dark grey in transmission, violet in reflection) due to coupling between the dimer disks. Inset: SEM image of the nanodisk dimers before deposition of TCO.

stage was used to produce a variable delay up to several hundred picoseconds. The picosecond tunable output of the supercontinuum was used as a probe for detecting small changes in the detected light intensity due to the presence of the pump laser. To recover the small modulated components, the pump light was modulated at $1.2 \mathrm{kHz}$ using a mechanical chopper, and the modulated intensity was detected using a DSP lock-in amplifier (Scitec).

\section{Results}

The linear spectral response of the plasmonic antenna layer without TCO coverage is shown in Fig. 2. The presence of the plasmonic dimers is observed as a dip both in the reflection and the transmission spectra. The longitudinal response of the particle dimers is redshifted in comparison to the transverse one due to coupling between the disks of each dimer. The coverage with the various TCO layers leads to further redshift of the resonances as is shown in Fig. 3(a,b) showing the longitudinal polarization component in reflection. While the antenna layers on $\mathrm{SiO}_{2}$ show a reflectivity away from the resonance 
of around $4 \%$, the reflectivities of the ITO and AZO covered layers are around $6-8 \%$, which can be explained by the higher refractive index of these top layers $(n=2.0$ for pure $\mathrm{ZnO}$ ). For comparison, we also show the reflectivity of pure ITO and AZO films grown on glass substrates during the same fabrication runs but without nanoantennas. The antenna resonance results in a reduced reflectivity, apart from the long-wavelength range for the AZO sample, which will be discussed further below. The reflectivity of the antenna arrays consists of a mixing between the scattered fields of the antennas and the reflectivity of the substrate, known as interferometric scattering [18] and given by the relation

$$
R=\left|E_{\text {subs }}\right|^{2}+\left|E_{\text {scat }}\right|^{2}+2 E_{\text {subs }} E_{\text {scat }} \cos \phi,
$$

where $E_{\text {subs }}$ denotes the reflected field of the substrate, $E_{\text {scat }}$ the reflected field of the nanoantenna layer, and $\phi$ the relative phase difference between the two components. Generally, the mixing term dominates the response for weakly scattering particles $\left|E_{\text {scat }}\right|<\left|E_{\text {subs }}\right|$. The overall sign of the mixing term is determined by the relative phases of $E_{\text {scat }}$ and $E_{\text {subs. }}$. In the configuration where light is incident through the substrate side, $E_{\text {subs }}=r_{21} E_{\text {in }}$, where $r_{21}$ is the field reflection coefficient from glass to air. For incidence on the air-glass interface, $E_{\text {subs }}=r_{12} E_{\text {in }}$. Fresnel's equations for reflection show that $r_{21}=-r_{12}$, resulting in a sign change of the mixing signal in Eq. 2 for incidence from the air and glass side, respectively. This effect is shown in Fig. 3(e) where we have plotted the reflectivities for illumination of the antennas from the airglass interface (dashed line) and through the glass substrate (solid line). Indeed, a peak corresponding to the antenna resonance is observed for air-side illumination as reported in Ref. [13] while a dip is found for the glass-side illumination.

Nonlinear pump-probe spectra of the antenna-ITO and AZO hybrids are shown in Fig. 3(c,d). Similar to the response of a single nanoantenna [7], the total signal consists of a fast, picosecond contribution and a stationary component due to heat pileup in the sample over many laser pulses. In addition, Fig. 3(e,f) shows the response of the gold nanoantenna array without TCO cover layer. In absence of a TCO layer, the gold shows a typical plasmon bleaching response, characterized by a reduction of the peak absorbance and a broadening of the resonance profile [cf. Fig. 3(e,f)]. The signals for antennas covered with ITO and AZO in Fig. 3(c,d) are both dominated by a bipolar response with a zero crossing near or at the location of the minimum of the reflectivity curve. The strong similarity between the stationary and fast components indicates that this effect is due to a refractive index change of the TCO layer.

The ultrafast dynamics of colloidal gold nanorods and their cooling to a surrounding glass matrix has been studied intensively in recent years [19, 20]. Nonlinear changes in the extinction coefficient of a metal nanoparticle induced by ultrafast laser heating can be decomposed into the nonlinearity of both the metal and its surroundings according to $[20]$

$$
\Delta \sigma=\left(\frac{\partial \sigma}{\partial \epsilon_{1}} \frac{\partial \epsilon_{1}}{\partial T_{p}}+\frac{\partial \sigma}{\partial \epsilon_{2}} \frac{\partial \epsilon_{2}}{\partial T_{p}}\right) \Delta T_{p}+\left(\frac{\partial \sigma}{\partial \epsilon_{m, 1}} \frac{\partial \epsilon_{m, 1}}{\partial T_{m}}+\frac{\partial \sigma}{\partial \epsilon_{m, 2}} \frac{\partial \epsilon_{m, 2}}{\partial T_{m}}\right) \Delta T_{m}
$$



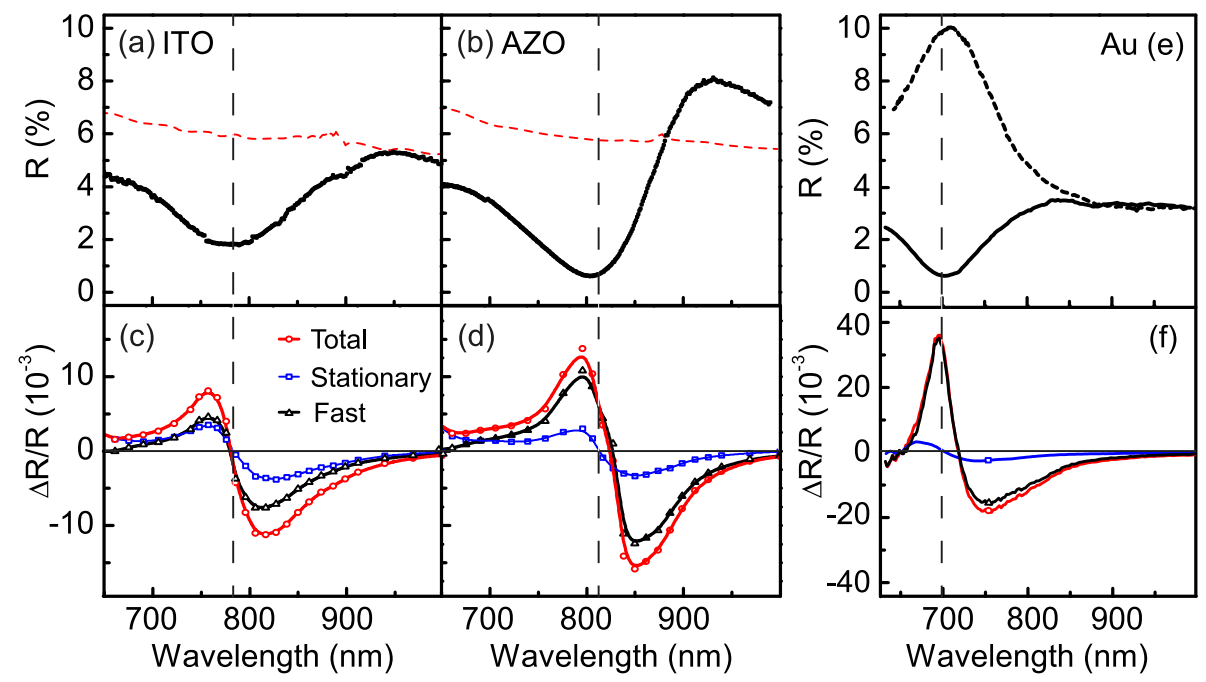

Figure 3. Reflectivity and nonlinear spectral response for antenna-TCO hybrids using ITO $(\mathrm{a}, \mathrm{c})$ and AZO $(\mathrm{b}, \mathrm{d})$. Dashed lines in $(\mathrm{a}, \mathrm{b})$ are reflectivities of corresponding TCO films without antennas. For the nonlinear signal, we differ between 3 components: the stationary heat signal (blue squares), the fast pump probe signal on top of the heat signal (red circles), and the effective difference between both (black triangles). (e,f) Same for a sample of Au nanoantennas without cover layer.

where $\tilde{\epsilon}=\epsilon_{1}+i \epsilon_{2}$ is the dielectric function of the metal and $\tilde{\epsilon}_{m}=\epsilon_{m, 1}+i \epsilon_{m, 2}$ that of the host medium. $T_{p}$ and $T_{m}$ denote the temperatures of respectively the particle and the host medium. Our results on gold nanoantennas without cover layer are consistent with literature [19], where it was found that, for delay times $>4 \mathrm{ps}$, the response of the gold nanorod is governed by an imaginary component due to the modification of the Drude damping by lattice heating. On short $(<4 \mathrm{ps})$ timescales, interband excitations contributed a change in the real permittivity $\epsilon_{1}$ [19]. Our results do not show this shorttime component mainly because of the picosecond width of our pump pulses, which result in a more equilibrium heating and smearing out of fast components. In addition, the excitation wavelength of $530 \mathrm{~nm}$ is slightly below the interband absorption edge of $\mathrm{Au}$, therefore heating takes place through ohmic dissipation of surface plasmons, rather than through interband excitations. At long times $(t \approx 50 \mathrm{~ns})$, only a small stationary signal is found which has a bipolar shape with a zero crossing at the maximum of the resonance. This contribution can be attributed to a change in the refractive index of the glass substrate resulting from the pileup of heat generated by the gold nanoantennas.

The linear and nonlinear reflectivity of the antenna-TCO hybrids in Fig. 3 can be qualitatively understood using a simple multilayer model including the substrate, the nanoantenna-TCO hybrid array, and a TCO top layer. The refractive indices of substrate and top layer were taken as $n_{1}=1.5$ and $n_{3}=2.0$, respectively. The antennaTCO hybrid was modelled through a Maxwell-Garnett effective medium description, where we took an antenna volume fraction of $8 \%$ [13]. The longitudinal SPR of the nanoantennas was included using Mie-Gans theory. Figure 4(a,b) shows the calculated reflectivity spectra for the multilayer for various values of the TCO top layer thickness of 

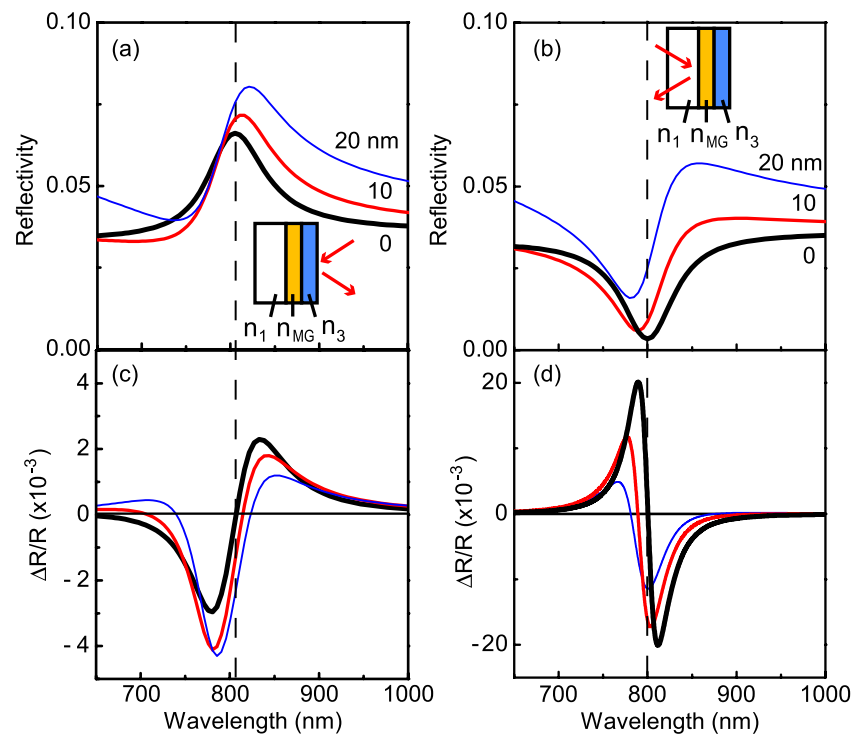

Figure 4. Reflectivity and nonlinear spectral response calculated using a multilayer model including a Maxwell-Garnett effective medium description of the Au-hybrid layer, for air-side $(\mathrm{a}, \mathrm{c})$ and glass-side $(\mathrm{b}, \mathrm{d})$ incidence. Calculations were done for a thickness of the TCO top layer of $0 \mathrm{~nm}$ (thick line, black), $10 \mathrm{~nm}$ (line, red) and $20 \mathrm{~nm}$ (thin line, blue). Insets in (a,b) show schematic of multilayer model with direction of incident light.

0, 10 and $20 \mathrm{~nm}$. The presence of a top layer results in an additional subtle change in the spectrum caused by the phase offset between the reflections from the antenna array and the TCO top-layer, resulting in an asymmetric Fano lineshape. The role of the phase offset can be understood by looking at the role of $\phi$ in Eq. 2, which essentially mixes in-phase and out-of-phase components in the reflected fields. We find good agreement with the shape of the measured reflectivity spectra in Fig. 3 for thicknesses in the range 10-20 nm for both the ITO and AZO samples.

Next, nonlinear reflectivity changes were calculated in response to a small change in the real part of refractive index of the host medium, $\Delta n_{m, 1}=10^{-3}$, where $\Delta n_{m, 1}=$ $\operatorname{Re}\left[\left(\tilde{\epsilon}_{m}\right)^{1 / 2}\right]$. The refractive indices of substrate and top layer were kept constant for the sake of simplicity. Figure $4(\mathrm{c}, \mathrm{d})$ shows the resulting reflectivity changes $\Delta R / R$ for the three values for the top-layer thickness under study. For a top-layer thickness of $0 \mathrm{~nm}$, we find a bipolar shape indicating a redshift of the antenna resonance. For glassside incidence in Fig. 4(d), the bipolar shape is preserved when increasing the top-layer thickness. The zero crossing shows a spectral shift which agrees with the shift of the spectral minimum in Fig. 4(b). For air-side incidence, a more complex evolution of the nonlinear spectrum is found. While the zero-crossing of $\Delta R / R$ in Fig. 4(c) still shifts with the peak of the reflectivity curve, an additional phase inversion appears at shorter wavelengths, corresponding to the negative lobe of the Fano-lineshape in reflectivity. These simple calculations show that nonlinear reflectivities for a multilayer structure strongly depend on the exact geometry. Our results of Fig. 3(c,d) agree qualitatively with the calculations for glass-side incidence using only a change in the real 


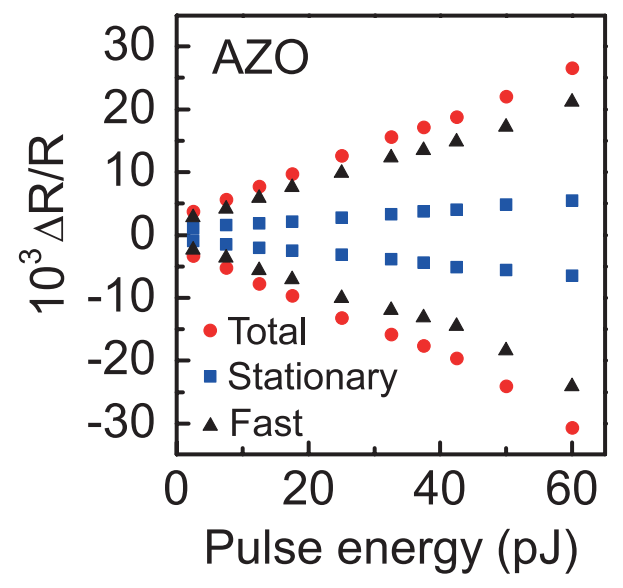

Figure 5. Dependence of the slow heat component (blue squares), the fast component (red circles) and their difference (black triangles) of $\Delta R / R$ on pump energy, at the peak positions of the dipolar signal for AZO at $795 \mathrm{~nm}$ and $850 \mathrm{~nm}$.

refractive index of the host medium, supporting our hypothesis of the hybrid antennaTCO response.

We proceed by further analyzing the properties of the antenna-TCO response found in Fig. 3(c,d). Figure 5 shows the dependence of $\Delta R / R$ on the intensity of the pump laser. Here, a $60 \mathrm{pJ}$ energy corresponds approximately to a fluence of $1.9 \mathrm{~mJ} / \mathrm{cm}^{2}$. Both the fast and stationary contribution show a linear dependence on pump intensity, confirming that our experiments are in the linear single-photon pumping regime (i.e. as opposed to two-photon excitation). The modulation amplitude of the reflected light is slightly lower, but of the same order of magnitude as values obtained for individual antenna-ITO hybrids, when we normalize the modulation amplitude to the spectral maximum of the antenna-induced reflectivity (i.e. Fig. 4 in Ref. [7]). Difference may be attributed to the different size of the antennas used, i.e. $200 \mathrm{~nm}$ in Ref. [7], and the concomitant larger amount of absorbed energy, and to differences in the carrier density of the TCO layers.

Time-resolved reflectivity spectra versus wavelength are shown in Fig. 6 for both ITO and AZO hybrids and for longitudinal and transverse polarizations. The thermal background signals have been subtracted for displaying purposes. Cross sections of the maps are shown in the right-hand panels of Fig. 6 for three wavelengths corresponding to the maximum, minimum, and zero crossings of the nonlinear signals. The time dynamics of the nonlinear response for both samples has been fitted to a biexponential decay as shown by the dashed green lines in Fig. 6. Resulting values for the decay times and amplitudes of the fast and slow components are shown in Table 1.

A large difference is observed in the dynamics of the nonlinear signal. The decay of the antenna-ITO signal is governed by a slow decay over 709 ps, with only a small component of time constant 82 ps. The antenna-AZO hybrid shows a much faster initial decay over $21 \mathrm{ps}$, followed by a slow decay of 543 ps of roughly the same amplitude. We attribute this difference to different thermal conductivities for the ITO and AZO. For 

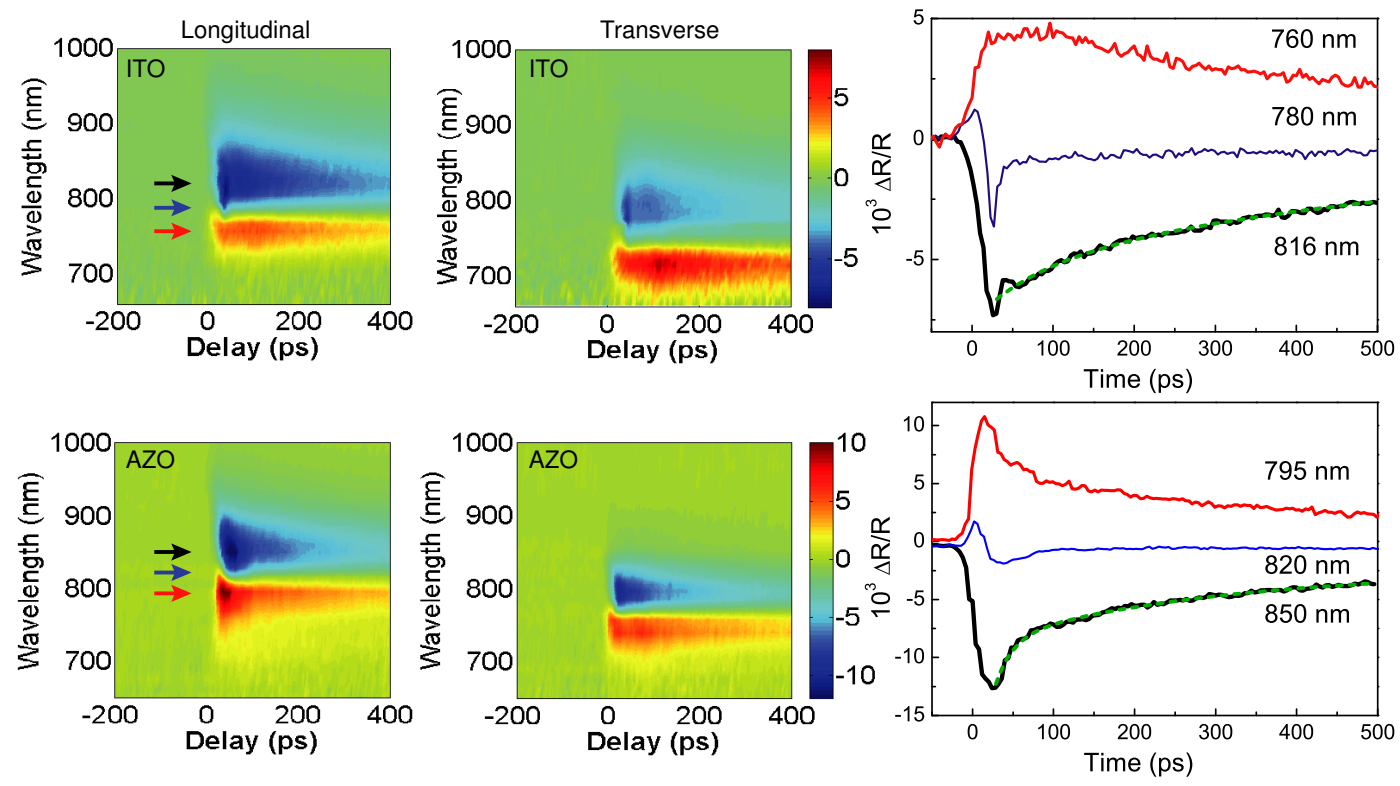

Figure 6. Time-resolved spectral maps of the pump-probe signals $\Delta R / R$ for both polarizations with the stationary heat component subtracted, both for dimers covered in ITO and AZO. (Right) Cross sections of $\Delta R / R$ versus time at three wavelengths corresponding to the spectra of Fig. 3. (Dashed lines, green) Fits using biexponential decay for ITO and AZO, yielding time constants and amplitudes shown in Table 1.

the bulk materials, values for ITO of around $\kappa_{\mathrm{ITO}}=5.9 \mathrm{~W} \mathrm{~m}^{-1} \mathrm{~K}^{-1}$ have been reported [21]. For AZO values differ enormously from $\kappa_{\mathrm{ITO}}=1000 \mathrm{~W} \mathrm{~m}^{-1} \mathrm{~K}^{-1}$ for single-crystal $\mathrm{ZnO}$ down to hundred times less for polycrystalline thin films. It is well known that for polycrystalline materials, the heat conductivity is limited by diffusion barriers over grain boundaries [23]. The high quality of the AZO deposited using ALD is likely to result in better heat conductivity properties than the polycrystalline sputtered ITO. Our results call for more systematic studies of the properties of the layers produced by ALD. The similarity of the slow decay component for both layers suggest that this contribution is associated to the cooling down of the thin-film oxide through the glass substrate.

Another characteristic feature in the time-resolved maps is the fast blueshift of the bipolar signal in the first tens of picosecond following the excitation. This transient response is clearly observed in the time traces around the zero crossing of the spectral profile (blue lines in Fig 6). The exact origin of this recurrent feature is not yet understood at the moment. The blueshift seems to be related to the nonequilibrium electron dynamics directly following the picosecond pump pulse. Alternatively, this fast transient response may be associated with the generation of a strain wavepacket in the 20-nm thin oxide layer with frequency components up to several hundred $\mathrm{GHz}$ [24]. The details of this interaction will be investigated in future studies. 


\section{Conclusion}

In conclusion, we have investigated the use of different transparent conducting oxides for activation of hybrid metamaterial devices. We have shown that the effect of fast electron injection from plasmonic structures into their immediate surroundings is a very general effect that occurs in a variety of materials. Transparent conducting oxides such as ITO and AZO form a group of promising nonlinear materials for use in hybrid metamaterial optoelectronic devices. The availability of highly controllable fabrication methods using Atomic Layer Deposition make AZO an ideal candidate for device applications. The possibility to achieve modulation with a $20-\mathrm{nm}$ film as demonstrated here is a prerequisite for using this deposition technique which is limited to relatively thin layers. Further optimization may provide even more sizeable changes in refractive index and therefore nonlinear response of the hybrid devices. Tunable metamaterial devices using antenna-ITO hybrids will be of interest for a range of devices including spectral filters, active control of emitters and spasing, nonlinear optical wave conversion, and plasmonic biosensors.

O. L. M. acknowledges support from EPSRC through Grant EP/J011797/1. B. S. acknowledges financial support from the "Ramón and Cajal" program from the Spanish Ministry of Science and Innovation.

\section{References}

[1] A. Boltasseva and H. Atwater, Science 331, 290-291 (2011).

[2] P. R. West, S. Ishii, G. V. Naik, N. K. Emani, V. M. Shalaev, and A. Boltasseva, Laser Phot. Rev. 4, 795-808 (2010).

[3] K.F. MacDonald, N.I. Zheludev, Laser Phot. Rev. 4, 562567 (2010).

[4] M. W. Knight, H. Sobhani, P Nordlander, and N. J. Halas, Science 332, 702 (2011).

[5] F. Wang and N. A. Melosh, Nano Lett., 11, 54265430 (2011).

[6] S. Mubeen, G. Hernandez-Sosa, D. Moses, J. Lee, and M. Moskovits, Nano Lett. 11, 55485552 (2011).

[7] M. Abb, P. Albella, J. Aizpurua, O. L. Muskens, Nano Lett. 11, 2457-2463 (2011).

[8] K. Nomura, H. Ohta, A Takagi, T. Kamiya, M. Hirano, and H. Hosono, Nature 432, 488-492 (2004).

[9] Ramamoorthy Ramesh and Darrell G. Schlom, MRS Bulletin, 33, 1006-1014 (2008).

[10] T. Dekorsy, T. Pfeifer, W. Kütt, and H. Kurz, Phys. Rev. B 47, 38423849 (1993).

[11] H. Fredriksson, Y. Alaverdyan, A. Dmitriev, C. Langhammer, D. S. Sutherland, M. Zäch, and B. Kasemo, Advanced Materials, Vol. 19, Issue 23 (2007).

[12] T. Shegai, S. Chen, V. D. Mijković, G. Zengin, P. Johansson and M. Käll, Nat. Comm. 2, 481 (2011).

\begin{tabular}{|c|c|c|c|c|} 
& $t_{1}(\mathrm{ps})$ & $10^{3} A_{1}$ & $t_{2}(\mathrm{ps})$ & $10^{3} A_{2}$ \\
\hline ITO & $82 \pm 16$ & $-1.5 \pm 0.2$ & $709 \pm 53$ & $-5.1 \pm 0.3$ \\
\hline AZO & $21 \pm 1$ & $-5.1 \pm 0.2$ & $543 \pm 13$ & $-7.9 \pm 0.1$ \\
\hline
\end{tabular}

Table 1. Results from biexponential fits to Fig. 6, showing decay times $t_{1,2}$ and amplitudes $A_{1,2}$ of fast and slow components. 
[13] M. A. Otte, M.-C. Estévez, L. G. Carrascosa, A. B. González-Guerrero, L. M. Lechuga, and B. Sepúlveda, J. Phys. Chem. C 115, 53445351 (2011).

[14] S. Cataldo, J. Zhao, B. Frank, F. Neubrech, C. Zhang, P. V. Braun, and H. Giessen ACS Nano 6, $979(2012)$

[15] A. Janotti and C. G Van de Walle, Rep. Prog. Phys. 72, 126501 (2009).

[16] P. Banerjee, W.-J. Lee, K.-R. Bae, S. B. Lee, F. W. Rubloff, J. of Appl. Phys. 108, 043504 (2010).

[17] D.-J. Lee, H.-M. Kim, J.-Y. Kwon, H. Choi, S.-H. Kim, K.-B. Kim, Adv. Funct. Mater. 21, 448-455 (2011).

[18] P. Kukura, H. Ewers, C. Müller, A. Renn, A. Helenius, and V. Sandoghdar, Nat. Methods 6, 923-927 (2009).

[19] H. Baida, D. Mongin, D. Christofilos, G. Bachelier, A. Crut, P. Maioli, N. Del Fatti, and F. Vallée, Phys. Rev. Lett. 107, 057402 (2011).

[20] V. Juvé, M. Scardamaglia, P. Maioli, A. Crut, S. Merabia, L. Joly, N. Del Fatti, F. Vallée, Phys. Rev. B 80, 195406 (2009).

[21] T. Ashida, A. Miyamura, N. Oka, Y. Sato, T. Yagi, N. Taketoshi, T. Baba, and Y. Shigesato, J. Appl. Phys. 105, 073709 (2009).

[22] D. I. Florescu, L. G. Mourokh, Fred H. Pollak, D. C. Look, G. Cantwell, and X. Li, J. Appl. Phys. 91, 890-893 (2002).

[23] S.-M. Lee, D. G. Cahill, T. H. Allen, Phys. Rev. B 52, 253257 (1995).

[24] T. C. Zhu, H. J. Maris, and J. Tauc, Phys. Rev. B 44, 42814289 (1991). 